\title{
Manifestazioni extrarenali nella malattia renale policistica autosomica dominante
}

\author{
A. Sessa, F. Conte, P. Serbelloni, S. Milani \\ Unità Operativa di Nefrologia e Dialisi - Ospedale di Vimercate (MI)
}

e moderne tecniche diagnostiche quali l'ultrasonografia, la tomografia assiale computerizzata e la risonanza magnetica dell'addome, hanno chiaramente documentato come la presenza di cisti renali, singole o multiple, mono-bilaterali, siano un evento assai frequente, soprattutto nella popolazione anziana. In particolare, l'indagine ecografica è largamente impiegata per qualsiasi sintomo clinico d'origine addominale, e si è rivelata la metodica diagnostica più applicata per accertare patologie cistiche a carico d'organi parenchimatosi.

Le malattie cistiche renali possono essere acquisite o ereditarie; le forme ereditarie sono autosomiche e possono essere recessive o dominanti. Tra le forme autosomiche dominanti sono stati clonati i geni di tre malattie cistiche sistemiche: la malattia di Von-Hippel-Lindau, il complesso della sclerosi tuberosa, la malattia policistica autosomica dominante (Autosomal Polycystic Kidney Disease = ADPKD) (1). E a tutti nota l'eterogeneità del quadro clinico di ADPKD; le differenze fenotipiche tra i diversi pazienti possono essere, almeno in parte, spiegate dai diversi genotipi che caratterizzano questa malattia sistemica, dal momento che la patologia cistica è determinata, con ereditarietà autosomica dominante, da almeno tre differenti geni (PKD1, PKD2, PKD3) (1).

Qualsiasi sia l'errore genetico di cui è portatore un soggetto, la fenotipia che osserviamo in clinica è così complessa che risulta praticamente impossibile fare una diagnosi clinica dell'errore genetico presente in quel dato paziente e nella famiglia di sua appartenenza; è noto, infatti, che la trasformazione renale cistica è presente in tutti i pazienti, sia PKD1 sia non PKD1, e che il decorso clinico può essere estremamente variabile anche tra individui affetti appartenenti alla stessa fratria. Allo stesso modo, le manifestazioni patologiche extrarenali che sono state descritte, sono variamente rappresentate in pazienti sia PKDl, che non PDK1 (2).

Il termine "malattia epatica policistica" (MEP) è usato per descrivere la presenza di cisti multiple disseminate nel parenchima epatico, usualmente riscontrate in associazione con ADPKD, sia nei pazienti PKD1 che non PKD1 (3).

Non è certa l'esistenza di una malattia cistica epatica autosomica dominante, e non è probabile che soggetti appartenenti a famiglia con ADPKD presentino MEP in assenza di cisti renali. Inoltre, la possibilità che un paziente presenti cisti epatiche "isolate" in assenza di ADPKD ovviamente esiste, ma, spesso, si tratta di quadri clinici rari e di diversa natura patogenetica (dilatazione intraepatica o extraepatica dei dotti biliari, fibrosi epatica congenita, dilatazione segmentaria dei dotti biliari intraepatici - malattia di Caroli -, malattia renale politicistica autosomica recessiva).

La storia naturale della MEP è stata de- scritta in molti pazienti con ADPKD (2). $\mathrm{La}$ frequenza delle cisti epatiche incrementa con l'età: rare nei bambini, presenti nel $20 \%$ dei pazienti alla fine della terza decade, nel $75 \%$ nella settima decade di vita, e nel $100 \%$ dei pazienti sottoposti, con successo, a trapianto renale da più di dieci anni (4). La maggior parte dei pazienti, anche in presenza di diffusa e multipla MEP, sono asintomatici ed hanno normalità dei tests di funzionalità epatica. Le femmine presentano cisti epatiche più numerose, di dimensioni maggiori, in età più precoce, rispetto al sesso maschile, molto verosimilmente per ragioni ormonali; infatti, le donne che non hanno usato farmaci estro-progestinici e/o non hanno mai avuto gravidanze, presentano MEP meno conclamata rispetto a quelle che hanno avuto gravidanze o hanno fatto uso di estro-progestinici.

L'incidenza di MEP nei pazienti con $\mathrm{ADPKD}$, così come il numero ed il volume delle cisti epatiche, non si correlano con l'entità della compromissione della funzione renale (2). In presenza di coliche addominali o di dolori addominali persistenti, o di evidente versamento ascitico, il clinico deve prendere in considerazione la comparsa di complicazioni della patologia epatica, quali rotture di cisti, o emorragia intracistica, o infezione intracistica. L'infezione di una singola cisti epatica, oltre alla sintomatologia dolorosa, comporta iperpiressia, leucocitosi, positività di emocoltura e di coltura 


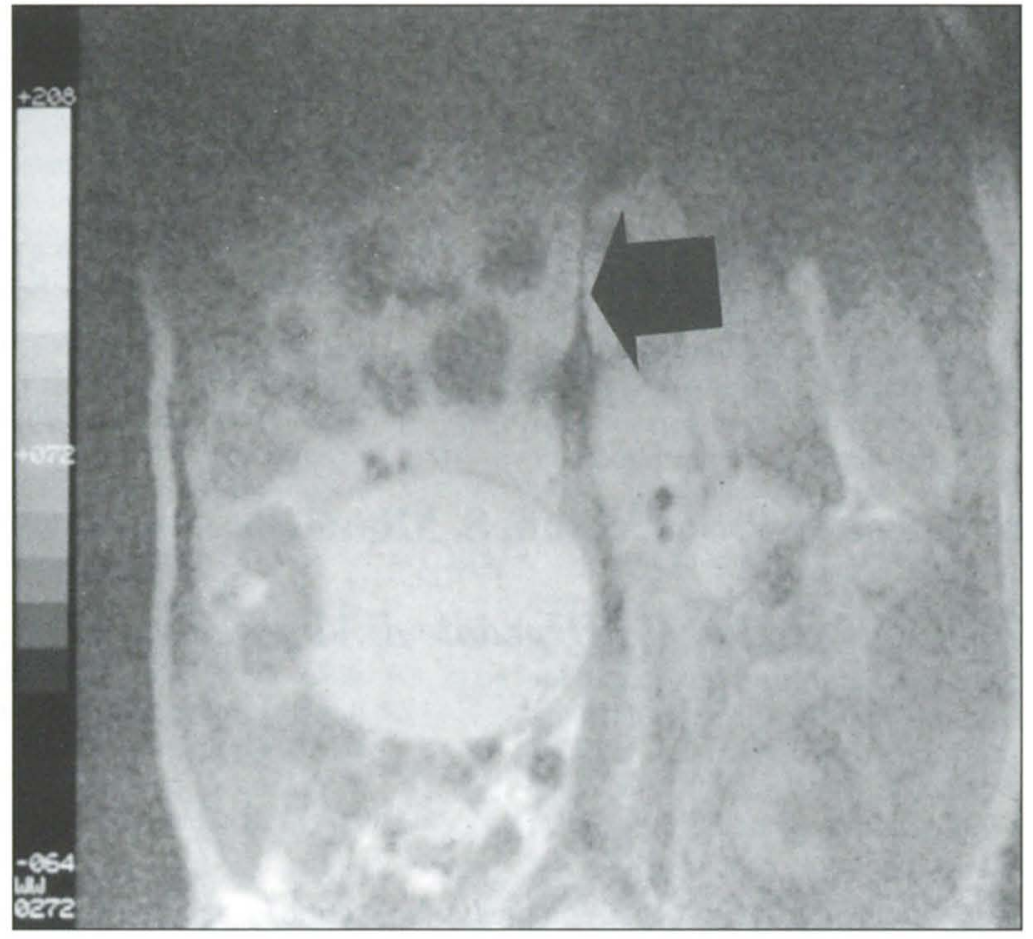

del liquido intracistico, incremento di tutti gli indici infiammatori; la metodica diagnostica più sensibile è la risonanza magnetica addominale.

Mentre in presenza di sintomatologia dolorosa addominale, anche se severa, ascrivibile alla patologia cistica epatica, è consigliato solamente un trattamento farmacologico analgesico perché, usualmente, la sintomatologia soggettiva è facilmente controllata in pochi giorni, in presenza di cisti infette occorre impiegare alti dosaggi di antibiotici che abbiano una particolare capacità di concentrazione nelle vie biliari, e può diventare mandatorio un drenaggio percutaneo e/o chirurgico della cisti infetta. L'ittero ostruttivo, quale complicazione determinata dalla compressione cistica sulle vie biliari, così come il versamento ascitico per compressione sulla vena porta, sono eventi estremamente rari (3). Alcuni pazienti hanno invece sviluppato una sindrome da ostruzione del flusso venoso intraepatico, secondaria a compressione intraepatica della vena cava, con versamento ascitico che può essere risolto soltanto con intervento chirurgico di epatectomia parziale e fenestrazione cistica (5) (Fig. 1).

Altri pazienti hanno sviluppato una sindrome di Budd-Chiari, a seguito di nefrectomia monolaterale o bilaterale di voluminosi reni policistici quale intervento in preparazione al trapianto di re- ne, a motivo di scivolamento del fegato cistico verso il basso e dall'indietro in avanti, con ostruzione e/o trombosi della vena cava inferiore o delle vene sovraepatiche (6).

Generalmente, i sintomi clinici di MEP sono ascrivibili a, e compaiono per, una massiva trasformazione cistica del fegato e sono correlabili all'effetto di ingombro addominale, con conseguente ortopnea, dispnea e, a volte, fratture costali; malnutrizione per precoce senso di sazietà, ernie addominali, prolasso utero-vaginale (3). In alcuni casi è necessario intervenire chirurgicamente con epatectomia di segmenti epatici, combinata con fenestrazione di cisti; solo raramente è stato necessario il trapianto di fegato (7).

L'incidenza di cisti pancreatiche in pazienti ADPKD non è ancora stata ben definita; tuttavia, studi autoptici (8) e studi clinici basati sulla diagnostica ecografica (9) indicano che circa il 9\% dei pazienti adulti presentano anche cisti pancreatiche. Le cisti pancreatiche sono rare in età giovanile e sono di più frequente riscontro in pazienti ADPKD con più di 30 anni di età; tuttavia, è assai verosimile che la prevalenza possa essere anche maggiore del 9\% dei pazienti, dal momento che l'indagine ecografica, per motivazioni tecniche, quali la presenza di gas nello stomaco, non sempre riesce ad esplorare completamente il pancreas, specie nel suo tratto più distale (la coda).
Fig. 1 - Risonanza magnetica addominale che documenta una massiva degenerazione cistica del fegato, con evidente compressione della vena cava ed assottigliamento del suo lume in corrispondenza del tratto intraepatico (freccia).

Il sesso femminile sembra essere più predisposto, forse in relazione a fattori ormonali. Non è stato identificato un particolare segmento preferenziale del pancreas in cui sia più frequente la presenza di cisti, così come non è stata evidenziata una correlazione tra volume delle cisti pancreatiche e dimensioni dei reni policistici (9). Le cisti pancreatiche hanno un decorso clinico asintomatico; in presenza di dubbio clinico sulla natura delle cisti pancreatiche (tumori cistici, cisti idatidee, pseudocisti, ecc.), e di incertezza sulla loro possibile natura solida invece che liquida, è necessario completare l'indagine dell'addome con la tomografia assiale computerizzata o con la risonanza magnetica.

La diverticolosi del tratto intestinale è di riscontro assai frequente nei pazienti con ADPKD, sebbene in letteratura esistano pochi studi sistematici che ne documentino la coesistenza. La maggiore prevalenza di diverticolosi, rispetto ad una popolazione controllo, si assocerebbe anche ad un incrementato rischio di perforazione intestinale (10). Studi autoptici, eseguiti alcuni anni fa, descrivevano la prevalenza di diverticolosi nell' $83 \%$ dei pazienti emodializzati con ADPKD, rispetto al $32 \%$ dei controlli uremici per altre cause morbose, e rispetto al 38\% di una popolazione di controllo, confrontati per età e sesso (11). Certamente, l'incidenza di complicazioni addominali cau- 
sate da diverticolosi del colon è maggiore nei pazienti uremici con $\mathrm{ADPKD}$, così come le morti per complicazioni causate da diverticolosi intestinale nei pazienti sottoposti a trapianto renale sono più frequenti rispetto a quanto si riscontri in altre patologie uremizzanti $(10,12)$. Non è noto se la diverticolosi del colon sia correlata ad un'anomala espressione genetica propria del PKD, oppure sia da imputarsi a fattori non genetici quali, ad esempio, un'aumentata pressione endoaddominale. Sicuramente la policistina è espressa anche nelle cellule epiteliali presenti alla base delle cripte intestinali, ma non è ancora stato stabilito un suo ruolo nel meccanismo patogenetico della formazione di diverticoli (13). Le complicazioni delle diverticolosi, quali la diverticolite e la perforazione intestinale, richiedono un tempestivo trattamento medico e/o chirurgico, senza differenza di approccio diagnostico e terapeutico tra i pazienti con o senza ADPKD (14).

È nota una rilevante incidenza di anomalie cardiovascolari nei pazienti affetti da ADPKD, ed è stata descritta l'associazione tra anomalie cardiache congenite e malattie cistiche renali in senso lato (15). Tra le più controverse patologie cardiache correlate con ADPKD sono le anormalità valvolari che, secondo alcuni Autori, sarebbero particolarmente frequenti: il prolasso della valvola mitrale è stato descritto nel $26 \%$ dei pazienti adulti affetti da ADPKD, rispetto al $2 \%$ di una popolazione di controllo $(16,17)$. Inoltre, sono state riportate presenza di valvola aortica bicuspide, insufficienza aortica, degenerazione mixomatosa della valvola mitrale, rigurgito mitralico. Tuttavia, il dato clinico più rilevante è che le complicazioni cardiovascolari rappresentano la maggiore causa di morte dei pazienti con ADPKD sottoposti ad un trattamento sostitutivo (dialisi o trapianto) a motivo di uremia cronica terminale $(8$, 11). L'ipertensione arteriosa è il più importante fattore di rischio per le complicazioni cardiovascolari. È noto che l'ipertensione arteriosa interviene precocemente nei pazienti con ADPKD, in presenza di funzione renale ancora normale in oltre il $60 \%$ dei casi ed in circa il $18 \%$ dei bambini (18), con la caratteristica di avere un'attenuata caduta dei valori pressori durante le ore del sonno (19) (pazienti "non dippers") fatto che di per sé si correlerebbe con un aumentato rischio di complicazioni cardiovascolari (20).
Quindi, il ruolo potenziale della ipertensione di indurre danni agli organi bersaglio (cuore, vasi cerebrali, retina, rene e circolazione sistemica) è incrementato nei pazienti con ADPKD. Nei soggetti con ipertensione arteriosa essenziale è noto che l'ipertrofia ventricolare sinistra (IVS) rappresenta la risposta cardiaca agli elevati valori pressori $(21,22)$. I pazienti ipertesi affetti da ADPKD risultano presentare una significativa più alta frequenza di IVS rispetto ad una popolazione di controllo di soggetti ipertesi essenziali, nel $46 \%$ dei maschi versus il $20 \%$ e nel $37 \%$ delle femmine versus il $12 \%$; l'IVS sembra essere associata a più alti livelli di pressione sistolica e diastolica. Inoltre, è stata documentata un'associazione tra IVS con l'incrementato volume renale e con una maggiore compromissione della funzionalità renale. La incrementata prevalenza di IVS sembra essere correlata, soprattutto, a due importanti fattori: l'esordio precoce della ipertensione arteriosa nei pazienti con ADPKD (età media 31 anni) (23), ed un non ottimale controllo dei valori pressori nella maggior parte dei pazienti (150/90 $\mathrm{mmHg}$ ) (24).

È opportuno ricordare che l'IVS è stata riscontrata in circa il $23 \%$ dei pazienti con ADPKD ancora normotesi, e senza alcuna relazione con i valori pressori basali, con l'età, il peso corporeo, la quantità di sodio presente nella dieta, la funzione renale, ma forse in relazione con fattori genetici. Tra i pazienti ipertesi con ADPKD la presenza di IVS è più comune nei soggetti più anziani, con massa corporea aumentata, con valori pressori più elevati, con valori di ematocrito più bassi, e con funzione renale più compromessa (24).

Recenti valutazioni ecocardiografiche hanno documentato un'aumentata prevalenza dell'indice di massa del ventricolo sinistro (IMVS) nei soggetti giovani con ADPKD rispetto ad una popolazione di controllo (25), e questo data risulta correlato anche con $\mathrm{i}$ livelli dei valori pressori (26); chiaramente, IMVS aumentato è stato riscontrato in pazienti adulti con ADPKD ipertesi e con decremento della funzione renale. Un incrementato IMVS è un fattore prognostico negativo ed è associato, nei pazienti uremici cronici in trattamento dialitico, ad una maggiore incidenza di mortalità cardiaca (27).

È stato riportato che $\mathrm{i}$ bambini ipertesi con ADPKD presentano un IMVS au- mentato rispetto agli affetti che non hanno ipertensione arteriosa, e i bambini con ADPKD non ipertesi hanno un IMVS maggiore rispetto ai loro germani che non hanno ADPKD (25), a riprova del fatto che anche in assenza di ipertensione arteriosa questa malattia sistemica frequentemente coinvolge le strutture miocardiche.

Uno degli aspetti più allarmanti delle manifestazioni extrarenali di ADPKD è l'improvvisa rottura di un aneurisma intracranico. È stato riportato che la frequenza di aneurismi cerebrali nei pazienti con PKD1 è di circa il $10 \%$ (28), e la loro prevalenza è del $26 \%$ nei pazienti con ADPKD che presentano una storia familiare per aneurismi cerebrali (29). Il riscontro di aneurismi intracranici è stato riportato anche in pazienti appartenenti a famiglie PKD2 (30). Va osservato che pazienti con ADPKD hanno un incrementato rischio di sviluppare non solo gli aneurismi intracranici $(10,28,31)$, ma anche delle arterie cervicocefaliche (32), della aorta toracica $(33,34)$ ed addominale (35).

Recentemente è stata descritta anche la presenza di dolicoectasia (allungamento e dilatazione) di un segmento dell'arteria cerebrale, che può essere causa di emorragia cerebrale, e può radiologicamente mimare un aneurisma sacculare (36). L'interruzione della membrana elastica interna è stata documentata sia in corrispondenza di aneurismi che di dolicoectasie cerebrali, ed è stata riscontrata anche in arterie intracraniche normali di pazienti con ADPKD (37), facendo prendere in considerazione nel meccanismo patogenetico il ruolo specifico della policistina nel mantenere l'integrità delle matrici extracellulari (38) e, quindi, anche della muscolatura liscia delle arterie (39). La rottura di aneurismi cerebrali è stata riscontrata soltanto nel $25 \%$ dei pazienti con ADPKD che giungevano ad un ricovero ospedaliero a motivo di un evento neurologico acuto (40). La sede di rottura è più frequentemente localizzata nella arteria cerebrale mediana, ed avviene in corrispondenza di aneurismi di dimensioni maggiori ed in associazione ad ipertensione arteriosa (41).

In circa il $30 \%$ dei pazienti gli aneurismi cerebrali sono multipli e, quindi, il rischio di ricorrenza di accidenti neurologici acuti è elevato. Non sono stati identificati particolari "markers" clinici predittivi dell'esistenza di malformazioni vascolari intracraniche, né è stata docu- 


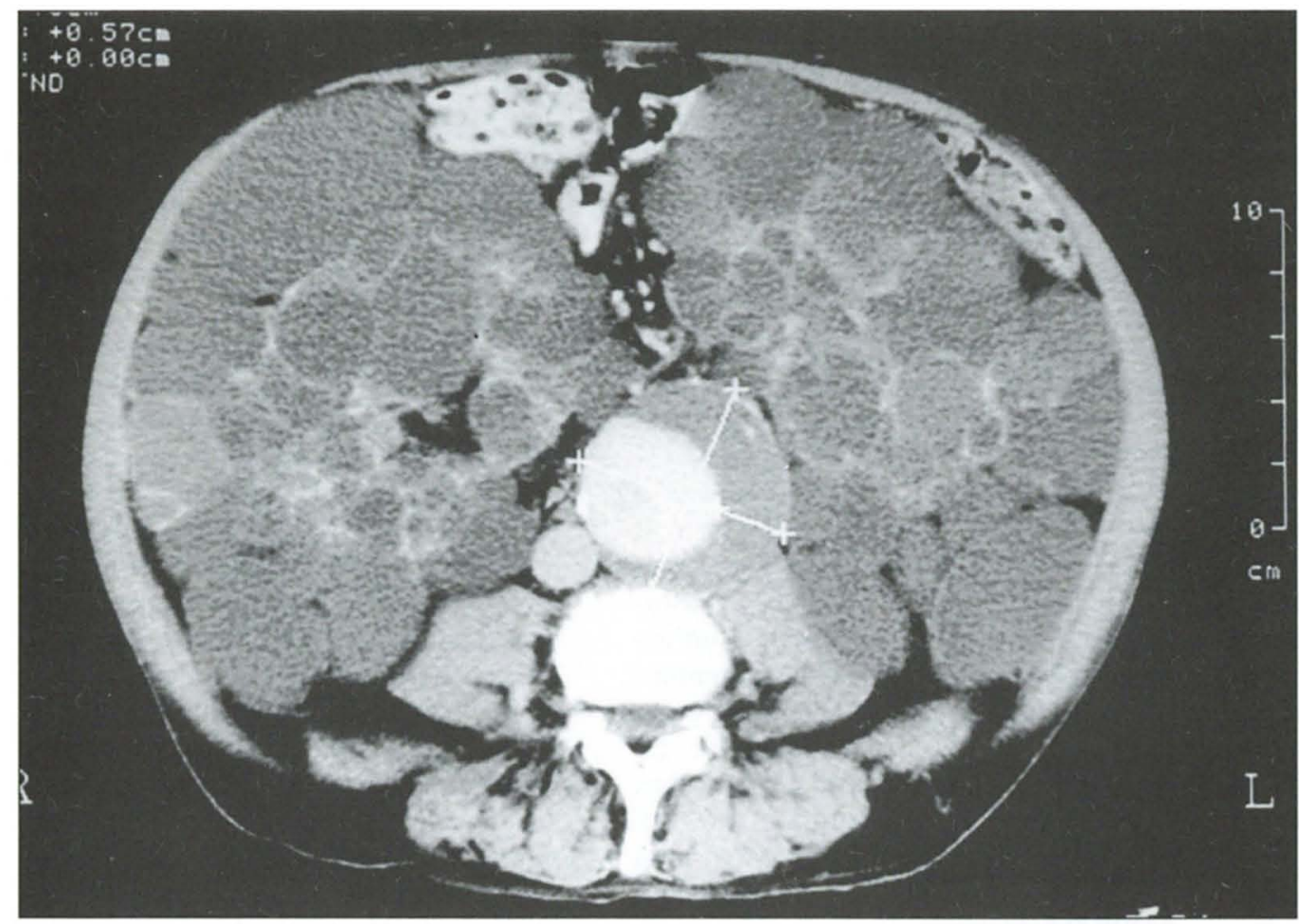

Fig. 2 - Ultrasonografia addominale. Evidenza di reni policistici bilaterali e di voluminoso aneurisma dell'aorta addominale.

mentata una specifica mutazione genetica per aneurismi cerebrali nei pazienti con ADPKD. Per tali motivi, non esiste l'indicazione di ricerca routinaria di aneurismi cerebrali, mediante la tecnica della angiorisonanza magnetica cerebrale, nei pazienti con ADPKD, a meno che presentino dei chiari sintomi clinici (severa ed inusuale cefalea), oppure si tratti di pazienti sopravvissuti ad una pregressa rottura di aneurisma intracranico, oppure appartengano a famiglie con evidenza di aneurismi cerebrali (42). Infatti, è stata documentata l'aggregazione familiare di aneurismi intracranici associata ad ADPKD (43), situazione clinica che deve essere tenuta distinta da un'altra condizione ereditaria rappresentata dagli "aneurismi intracranici familiari" (44).

È stato riportato che pazienti con ADPKD presentano una maggiore incidenza di dissezione della aorta toracica rispetto alla popolazione generale; tuttavia, la documentazione attualmente esistente è piuttosto esigua $(32,45,46)$. Poiché anomalie della matrice extracellulare con coinvolgimento del collagene di tipo IV, proteoglicani, fibronectina, tenascina, con anomalie della loro deposizione e dei loro reciproci rapporti sono state descritte nel rene policistico, non è escluso che la policistina possa svolgere un ruolo patogenetico nelle alterazioni istologiche di parete dell'aorta toracica nei pazienti con ADPKD (47).

Anche sulla reale incidenza di aneurismi a livello dell' aorta addominale in pazienti con ADPKD esistono dati contraddittori in letteratura (48). Ovviamente, rimane il dubbio se la presenza di aneurismi dell'aorta addominale sia semplicemente il risultato di una malattia renale cronica che comporta ipertensione arteriosa ad insorgenza giovanile, con le generiche complicazioni aterosclerotiche, oppure se in realtà esista una relazione specifica con ADPKD, a motivo delle sopraelencate anomalie della matrice extracellulare. Oggigiorno non esiste una relazione documentata tra le due patologie, anche se in letteratura sono riportati casi clinici con coincidenza delle due entità $(35,49)$. D'altra parte, è noto che la prevalenza di aneurismi dell'aorta addominale varia dal 4.3 al $8.8 \%$ della popolazione normale con più di 65 anni, con una maggiore incidenza nel sesso maschile. Gli aneurismi hanno una maggiore probabilità di rottura (5\% anno) quando raggiungono la dimensione di $5-6 \mathrm{~cm}$, con una probabilità esponenzialmente crescente con l'ingrandirsi dell'aneurisma, e con un rischio incrementato nei soggetti ipertesi (50).

Dal momento che la rottura di un aneurisma asintomatico dell'aorta addominale ed il suo trattamento di emergenza comportano un'alta incidenza di mortalità (51), è consigliabile completare con una valutazione dell'aorta addominale lo studio eccgrafico dell'addome in tutti i pazienti portatori di ADPKD, sia ipertesi che normotesi (Fig. 2). 
BIBLIOGRAFIA

1. Sessa A, Ghiggeri GM, Turco A. Autosomal dominant polycystic kidney disease: clinical and genetic aspects. J Nephrol 1997; 10: 295-310.

2. Gabow PA, Johnson AM, Kaeny WD, et al. Risk factor for the development of hepatic cysts in autosomal dominant polycystic kidney disease. Hepatology 1990; 11: 10337.

3. Torres VE. Polycystic liver diseases. In: Autosomal Dominant Polycystic Kidney Disease. A. Sessa, F. Conte, P. Serbelloni, S. Milani (Eds). Contr Nephrol 1995; 115: 4452. Inc Karger, Basel.

4. Fitzpatrick OM, Torres VE, Charbonneau JW, et al. Long-term outcome of renal transplantation in autosomal dominant polycystic kidney disease. Am J Kidney Dis 1990; 15: 535-43.

5. Torres VE, Rastogi S, King $\mathrm{BF}$, et al. Hepatic venous outflow obstruction in autosoma dominant polycystic kidney disease. J Am Soc Nephrol 1994; 5: 1186-92.

6. Dionisio P, Sessa A, Conte F, et al. Budd-Chiari syndrome following pretransplant mononephrectomy in ADPKD patient with liver cysts. Nephron 1997; 75: 109-11.

7. Que F, Nagorney DM, Gross $\mathrm{JB}$, Torres VE. Liver resection and cyst fenestration in the treatment of severe polycystic kidney disease. Gastroenterology 1995; 108: 484-94.

8. Fick GM, Johnson AM, Hammond WS, Gabow PA. Causes of death in autosomal dominant polycystic kidney disease. J Soc Nephrol 1995; 5: 2048-56.

9. Torra R, Nicolau C, Badenas $C$, et al. Ultrasonographic study of pancreatic cysts in autosomal dominant polycystic kidney disease. Clin Nephrol 1997; 47: 19-22.

10. Gabow PA. Autosomal dominant polycystic kidney disease. NEJM 1993; 329: 332-42.
11. Iglesias C, Torres V, Offord K, et al. Epidemiology of adult polycystic kidney disease. Olmstead country, Minnesota: 1935-1980. Am J Kidney Dis 1983; 2: 630-9.

12. Kaeny W, Everson G. Extrarenal manifestations of autosomal dominant polycystic kidney disease. Semin Nephrol 1991; 11: 661-70.

13. Griffin M, Torres V, Grande J, Kumar R. Immunolocalization of polycystin in human tissues and culturied cells. Proc Assoc Am Phys 1996; 108: 185-97.

14. Perrone RD. Extrarenal manifestation of ADPKD. Kidney Int 1997; 51: 2022-36.

15. Evans JA, Strane LC. Cystic renal disease and cardiovascular abnormalities. Am J Med Genet 1989; 33: 398-401.

16. Hossack KF, Leddy CL, Johnson AM, Schreir RW, Gabow PA. Echocardiographic findings in autosomal dominant polycystic kidney disease. NEJM 1988; 319: 907-12.

17. Timio M, Monarca C, Pede S, et al. The spectrum of cardiovascular abnormalities in ADPKD: A 10 years follow-up in a five-generation kindred. Clin Nephrol 1992; 5: 245-51.

18. Chapman AB, Gabow PA. Hypertension in autosomal dominant polycystic kidney disease. Kidney Int 1997; 52 (S-61): 571-3.

19. Likamwa TC, Macnicol AM, Watson ML. Ambulatory blood pressure in hypertensive patients with autosomal dominant polycystic kidney disease. Nephrol Dial Transpl 1997; 12: 2075-80.

20. Devereux RB, Pickering TG. Relationship between the level, pattern and variability of ambulatory blood pressure and target organ damage in hypertension. $\mathbf{J}$ Hypertens 1991; 9: (S-8): 534-8.

21. Verdecchia P, Schillaci G, Guerrier M. Correlation of blood pressure change and left ventricular hypertrophy in essential hypertension. Circulation 1990; 81: 81-9.
22. Koren MJ, Devereux RB, Casale PM, Savage DD, Laragh JH. Relation of left ventricular mass and geometry to morbidity and mortality in men and women with essential hypertension. Am Intern Med 1991; 114: $352-4$.

23. Gabow PA, Chapman AB, Johnson AM, et al. Renal structure and hypertension in autosomal polycystic kidney disease. Kidney Int 1990; 38: 1177-80.

24. Chapman AB, Johnson AM, Rainguet $S$, et al. Left ventricular hypertrophy in autosomal dominant polycystic kidney disease. J Am Soc Nephrol 1997; 8: 1292-7.

25. Ivy DD, Shaffer EM, Johnson AM, Kimberling WJ, Gabow PA. Cardiovascular abnormalities in children with autosomal dominant polycystic kidney disease. J Am Soc Nephrol 1995; 5: 2032-6.

26. Zeier M, Geberth S, Schmidt KG, Mandelbaum A, Ritz E. Elevated blood pressure profile and left ventricular mass in children and young adults with ADPKD. J Am Soc Nephrol 1993; 3: 1451-7.

27. Parfry PS, Griffiths SM, Harnett JD, et al. Outcome of congestive heart failure, dilated cardiomyopathy, hypertrophic, hyperkinetic disease and ischemic heart disease in dialysis patients. Am J Nephrol 1990; 10: 213-21.

28. Schievink WI, Torres VE, Piepgras DG, Wiebers DO. Saccular intracranial aneurysms in autosomal dominant polycystic kidney disease. J Am Soc Nephrol 1992; 3: 88-95.

29. Ruggeri PM, Poulos N, Masaryk TJ, et al. Occult intracranial aneurysms in polycystic kidney disease: screening with MR angiography. Radiology 1994; 191: 33-9.

30. Vandiic MA, Chang PC, Peters DJM, Breuning MM. Intracranial arterial dolichoectasia in polycystic kidney disease linked to chromosome 4. J Am Soc Nephrol 1995; 6: 1670-3.

31. Huston J III, Torres VE, Sullivan $\mathrm{PP}$, Offord KP, Wiebers DO. 
Value of magnetic resonance angiography for the detection of intracranial aneurysms in autosomal dominant polycystic kidney disease. J Am Soc Nephrol 1993; 3: 1871-7.

32. Somlo S, Rutecki G, Giuffra LA, et al. A kindred exhibitiing consegregation of an overlap connective tissue disorder and the chromosome 16 linked form to autosomal dominant polycystic kidney disease. J Am Soc Nephrol 1993; 4: 1371-8.

33. Swan SK, Kraemer MD, Henty TD, Collins AJ, Herzog CA. Increased incidence of coronary artery aneurysms with autosoma dominant polycistic kidney disease (ADPKD). J Am Soc Nephrol 1994; 5: 653-9.

34. Biermann CW, Gasser TC, Breuer C, Rutishauser G. Marfan syndrome and cystic kidneys of the adult type. Helv Chir Acta 1992; 59: 513-5.

35. Torra R, Nicolau C, Badenas $\mathrm{C}$, et al. Abdominal aortic aneurysms and autosomal dominant polycystic kidney disease. J Am Soc Nephrol 1996; 7: 2483-6.

36. Schievink WI, Chang PC, Peters DJM, Breuning MH. Intracranial arterial dolichoectasia in autosomal dominant polycystic kidney disease. J Am Soc Nephrol 1997; 8: 1298-303.

37. Ikeda H, Yochimoto T. Pathology study of Willi's circle in autopsy cases of polycystic kidney disease. No To Shinkei 1987; 39: 909 13.

38. The International Polycystic Kidney Disease Consortium. Polycystic kidney disease: The complete structure of the PKD1 gene and its protein. Cell 1995; 81: 28998.

39. Griffin MT, Torres VE, Grande JO, Kumar R. Vascular expression of polycystin in ADPKD and control subjects. J Am Soc Nephrol 1997; 8: 616-26.

40. Ryu SJ. Intracranial hemorrhage in patients with polycystic kidney disease. Stroke 1990; 21: 291-4.
41. Chauveau D, Pirson Y, Verellen DU, et al. Intracranial aneurysms in autosomal dominant polycystic kidney disease. Kidney Int 1994; 45: 1140-6.

42. Pirson Y, Chauveau D. ADPKD-associated intracranial aneurysms: new insights and unanswered questions. Contrib Nephrol 1995; 115: 53-8. Eds. A Sessa, F Conte, P Serbelloni, S Milani.

43. Lozano AM, Leblanc R. Cerebral aneurysms and polycystic kidney disease: a clinical review. Can J Neurol Sci 1992; 19: 222-7.

44. Ronkainem A, Hernesniemi J, Puranem M, et al. Familiar intracranial aneurysms. Lancet 1997; 349: 380-4.

45. Biagini A, Maffel S, Baroni $\mathrm{M}$, et al. Familiar Clustering of aortic dissection in polycystic kidney disease. Am J Cardiol 1993; 72: 741-2.

46. Hartman D. Autosomal dominant polycystic kidney disease complicated by thoracic aortic dissection. Am J Roentgenol 1994; 162: $1454-8$.

47. Paynter HE, Parnham A, Feest TG, Dudley GRK. Thoracic aortic dissection complicating autosomal dominant polycystic kidney disease. Nephrol Dial Transpl 1997; 12: 1711-3.

48. Vanmaele $\mathrm{R}$, Witbreuk $\mathrm{M}$, Debroe M, et al. Abdominal aortic aneurysm and polycystic kidney. Nephron 1995; 69: 107-8.

49. Stiasny B, Schulze BD, Graf S, Weber M. Vessel malformation in a patient with ADPKD: megadolichobasilar artery, abdominal aortic and iliac aneurysms. Contrib Nephrol 1995; 115: 182-4. Eds A Sessa, F Conte, P Serbelloni, S Milani.

50. Vandervliet JA, Boll APM. Abdominal aortic aneurysms. Lancet 1997 ; 349: 863-6.

51. Scott RAP, Ashton HA, Kay DN. Abdominal aortic aneurysm in screened patients: prevalence, development and management over 6 years. Br J Surg 1991; 78: 1122-5. 